\title{
Schwarz, verhüllend, weiblich. Die Inszenierung von Trauer und der Wandel von Gendervorstellungen
}

\author{
Anna-Katharina Höpflinger
}

Socio-religious bourgeois representations of mourning reached a climax in the 19th century. The traditions practiced around graves, the mourning attire, and the norms regulating ones behaviour were highly gendered: Mourning was - based on the idea of the emotional woman and the rational man - something deemed "naturally" female. However, these mourning practices have changed over the course of the 20th century. A strong stimulus for these changes has been the transformation of gender concepts, especially the collective expectations of the ideal woman. This paper discusses the transformations of socio-religious mourning representations on the basis of the idea of gender-changes.

Trauerkleidung; sozioreligiöse Transformationen; Gender und Trauern im 19. und 20. Jahrhundert; Tod, Religion und Gender

Mourning dress; socio-religious transformation; gender and mourning in the 19th and 20th century; death, religion, and gender

Anna-Katharina Höpflinger holds a Ph.D. in the Study of Religion and is a research and teaching assistant at the Ludwig-Maximilians-University in Munich. She has developed different research projects in the field of media and religion, particularly focusing on the body, clothing, and gender. Recent publications: ed. with Dolores Z. Bertschinger/ Natalie Fritz/ Marie-Therese Mäder: Augen-Blicke. 29 Perspektiven auf Religion (Zurich 2017); with Yves Müller: Ossarium. Beinhäuser der Schweiz (Zurich: 2016).

\section{Vom ,Leichenhuhn“" zum Internet: Einleitung}

1797 beschrieb J.J. Ludwig Geist, Goethes Schreiber, einen Leichenzug in der Schweiz:

Nun zieht denn alles in gehöriger Rangordnung hinter dem Sarg her, erst kommt der älteste Sohn des Verstorbenen oder auch die Tochter, die das Recht haben, neben der linken Seite des Sarges herzugehen, dann folgt die sämtliche Geistlichkeit und dann die übrigen Bürger, alle bar ${ }^{1}$, sodann folgen die Frauenzimmer, die aber nicht eher aus dem Haus der Leiche herausgehen als bis der Verstorbene fortgetragen wird. Die Anver-

1 Ohne Kopfbedeckung. 
Anna-Katharina Höpflinger

wandten ${ }^{2}$ sind alle schwarz mit weissen Mützen und mit einem schwarzen Schleyer bedeckt, in der Hand halten sie ein ganz weisses, viereckt zusammengelegtes Taschentuch. ${ }^{3}$

Diese Skizzierung eines Leichenzuges im späten 18. Jahrhundert zeigt, dass der sinnhafte Umgang ${ }^{4}$ mit Sterben und Tod nicht nur mit religiösen Vorstellungen und Praktiken verbunden ist, sondern auch Gendernormen berührt; denn es ist kein Zufall, dass die „Frauenzimmer“ bei dieser nach „gehöriger Rangordnung“ aufgestellten Leichenprozession ganz am Ende des Zuges angesiedelt werden. Der Umgang mit Sterben und Tod war in Europa - dem geographischen Fokus des vorliegenden Beitrags - jahrhundertelang genderisiert. ${ }^{5}$ Es ist auffallend, dass sich die sozioreligiösen ${ }^{6}$ Normierungen, die sich in der Neuzeit rund um den Tod etabliert hatten - Albert Hauser spricht von „traditionellen Praktiken“7 letzten Drittel des 20. Jahrhunderts besonders stark zu wandeln begannen. ${ }^{8}$ Armin Nassehi und Georg Weber haben deshalb von einer Verdrängung des Todes gesprochen $;{ }^{9}$ einer These, die der Komplexität des Wandels bezüglich des Umgangs mit dem Tod nicht gänzlich gerecht wird, denn es ist zu unterscheiden, auf welcher soziokulturellen Ebene sich der Umgang mit dem Tod wie ändert. ${ }^{10}$ Einerseits kann eine Verdrängung des Todes aus der Öffentlichkeit im Zug einer medizinischen Institutionalisierung und „Technisierung “11 des Umgangs mit dem Sterben, einhergehend mit einer Verlagerung der Toten in spezialisierte Räume beobachtet werden. Die öffentliche Aufbahrung des Leichnams findet sich v.a. noch in ruralen Gebieten, ${ }^{12}$ das gemeinschaftliche Herumtragen des Sarges im Rahmen einer Trauerprozession ist, bis auf die erwähnten großen Staatsbegräbnisse, nahezu ganz verschwunden. Trauerkleidung wird gegebenenfalls noch im Kontext der Bestattungsfeier angezogen, darüber hinaus sind aber viele vestimentäre Codes $^{13}$ für das Anzeigen eines Trauerstatus in der Alltagskleidung

2 Gemeint sind die anverwandten Frauen.

3 Zitiert nach Hauser 1994, S. 74.

4 „Sinnhafter Umgang“ wird im Folgenden verstanden als Handlungen und Praktiken zur Formung von sozialer Orientierung.

5 Vgl. Todd 1993; Goodwin 1993; Guthke 1998; Kneuper 1999; Ellwanger / Helmhold / Helmers / Schrödl 2010; Schroer 2014.

6 Ich verwende den Begriff „sozioreligiös“, um soziale Praxis, die von religiösen Normen, Vorstellungen und Handlungen geformt wird, zu bezeichnen.

7 Hauser 1994.

8 Vgl. Fischer 2001.

9 Vgl. Nassehi / Weber 1989.

10 Vgl. dazu die Aufsätze in Gehring 2007.

11 Vergleiche zu diesem Begriff und der Pragmatisierung des Todes durch die Kremation: Fischer 1996, S. 94-106.

12 Vgl. Höpflinger 2015; Höpflinger / Müller 2016.

13 Ich spreche hier absichtlich von Code und nicht von Zeichen oder Symbol. Ich 
verschwunden. Abb. 1 zeigt diesen Wandel stellvertretend am Beispiel einer Leichenkutsche (im Hintergrund), vermutlich aus dem frühen 20. Jahrhundert und eines gegenwärtigen Leichenwagens (im Vordergrund links). Letzterer ist in dezentem Grau gehalten und erst auf den zweiten Blick als Auto eines Bestattungsunternehmens erkennbar. Dieses Beispiel illustriert die Veränderung der öffentlichen Inszenierung von Tod.

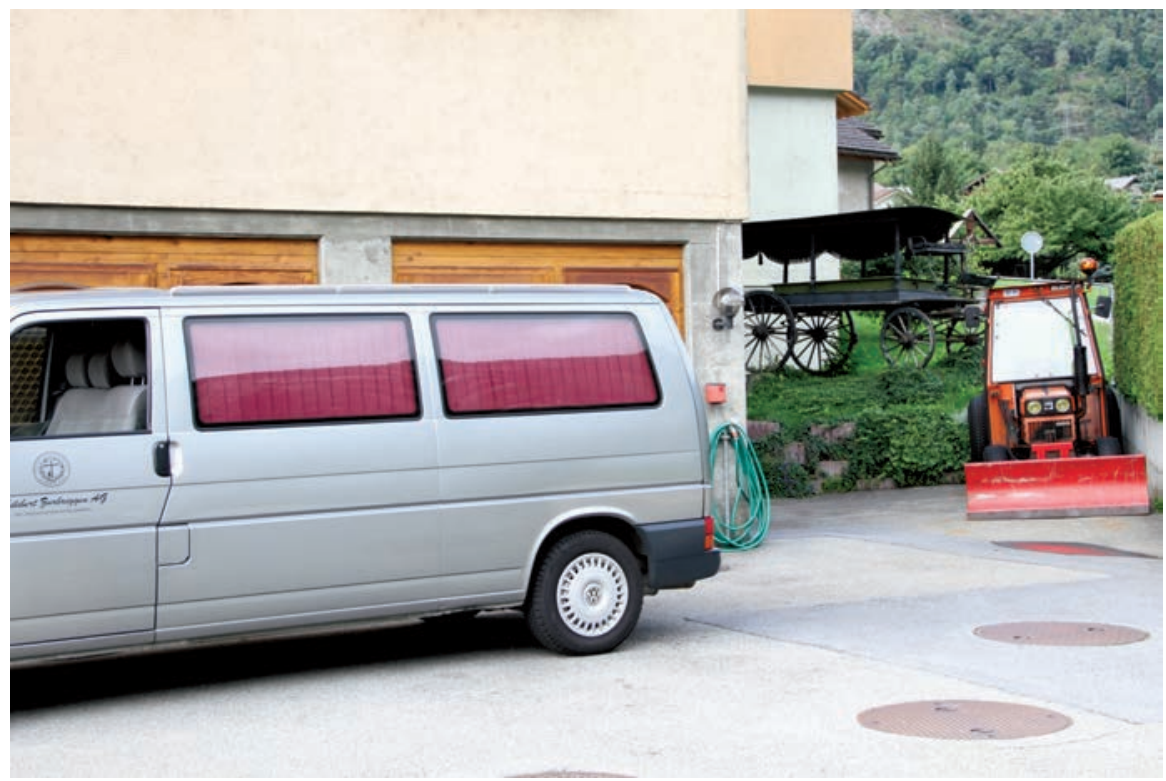

Abb. 1: Eine alte Begräbniskutsche (hinten, in Schwarz) zeugt von der Inszenierung des Todes; der gegenwärtige Leichenwagen (vorne links) wird dezent in Grau mit bordeauxroten Vorhängen repräsentiert. Bild aus Naters (VS, CH). (C) Yves Müller.

Andererseits wird Tod gegenwärtig massenmedial immer „nahegehender“ und „faktischer“ inszeniert und z. B. in Filmen, Liedern, Literatur, TV und im Internet rege thematisiert. ${ }^{14}$ Obwohl es auch heute noch grosse Staatsbegräbnisse und das gemeinsame Trauern bei öffentlichen Katastrophen wie Amokläufen und Terroranschlägen gibt, ist der sozioreligiöse Umgang mit dem weniger die Gesamtgesellschaft betreffenden Sterben also zunehmend aus dem öffentlichen in den massenmedialen Raum ausgelagert worden; die massenmediale Präsenz des Todes ändert nichts an der fehlenden Nähe zum Tod auf der praktischen Ebene. Unterdessen finden sich öffentlich-mediale Gegenbewegungen, die Death

umreiße Code als eine syntaktische Zusammenstellung aus Zeichen, die semantisch über sich selbst hinaus verweisen können, aber nicht müssen.

14 Vgl. Mäder 2012. 
Awareness Movements, die versuchen, den „faktischen“ Tod wieder in den Alltag zu integrieren. Ein prägnantes Beispiel hierfür ist der Order of the Good Death, der vor allem via social media, aber auch in Vorträgen präsent ist. ${ }^{15}$

Es ist also in den letzten 150 Jahren eine Veränderung der öffentlich-medialen Inszenierung von Tod und Trauer zu beobachten. Dieser Wandel ist multikausal: Dabei bedeutsam waren zunächst technische Erfindungen, die eine erweiterte Repräsentation des Todes in neuen Medien ermöglichten. So wurde beispielsweise der Beruf der (meist weiblichen) Leidansagerin, bzw. Leichenbitterin, die einen Todesfall ausrief oder ihn sogar von Haus zu Haus verkündete und manchmal despektierlich als „Leichenhuhn“ betitelt wurde,${ }^{16}$ in urbanen Gebieten spätestens in den 1930er Jahren durch gedruckte Todesanzeigen und heute bisweilen durch Meldungen im Internet ersetzt. In ruralen Regionen hielt sich diese Aufgabe bis ins ausgehende 20. Jahrhundert, verschwand dann jedoch ebenfalls. ${ }^{17}$ Noch wichtiger für die Änderung des Umgangs mit dem Tod war zweitens der medizinische Fortschritt, der das Sterben zunehmend ins Krankenhaus verschob, in dem auch das „Lagern“ der Toten professionalisiert wurde. ${ }^{18}$ Eine dritte zentrale Rolle bei diesem Wandel spielte, und das ist das Argument, das im folgenden Aufsatz verfolgt werden soll, eine Transformation der normativen Erwartungen gegenüber Genderrollen und ihrer öffentlichen Inszenierung. Ich will im Folgenden der Frage nachgehen, in welcher Relation der Wandel der Repräsentation von Trauer zur Veränderung mit Genderrollen verbundener sozioreligiöser Vorstellungen steht. Dabei werde ich, wie oben erwähnt, auf Europa und im weiteren Sinn auch auf die USA fokussieren und aus Platzgründen nicht einen historischen Überblick über Trauerpraktiken bieten, sondern diesen Wandel exemplarisch am offensichtlichsten Repräsentationscode von Trauer, nämlich der Kleidung, reflektieren. ${ }^{19}$ Ich werde dabei nur das 19. und 20. Jahrhundert in den Blick nehmen, die Zeit, in der sich dieser Wandel besonders stark ausprägt.

15 Vgl. Internetquellen: Order of the Good Death.

16 Es findet z. B. sich unter einem Stich aus der Stadt Zürich aus dem 19. Jahrhundert folgendes Gedicht: „In Zürich verkündet den Eintritt ins Leben / Ein Mädchen mit Bändern und Blumen geschmückt, / Doch hat der Tod die reife Frucht gepflückt / Und wird ein Mensch der Erde wieder gegeben, / So ruft ein Leichenhuhn, Gass' auf und ab, / Die Bürgerschaft an's offne Grab. / Der Künstler mag es wohl lieber halten / Mit jenem als mit der heulenden Alten." Vgl. zum Bild: Heierli 1930, Abb. 20 (Bildanhang).

17 Fischer 2001; Hauser 1994, S. 107. In meiner Jugend in den 1980er Jahren im ruralen Teil Graubündens $(\mathrm{CH})$ gab es dieses Amt noch: Eine Frau ging bei einem Todesfall von Haus zu Haus und meldete die Zeit der Beerdigung. Es gehörte zur dörflichen Pflicht, dass aus jedem Haushalt mindestens eine Person an der Bestattung anwesend war.

18 Vgl.: Fischer 2001; Hauser 1994 (für die Schweiz).

$19 \mathrm{Zu}$ Trauerkleidung vergleiche Taylor 1983; Hoefer 2010. 
Methodisch halte ich mich an ein kulturgeschichtliches Vorgehen und stütze mich sowohl auf textliche als auch auf ikonographische Quellen. ${ }^{20}$ Denn Todesund Trauerrituale werden nicht immer, wie oben bei J.J. Ludwig Geist, literarisch beschrieben, sondern oft bildlich dargestellt. ${ }^{21}$ Texte und Bilder wurden für den vorliegenden Aufsatz auf Gender fokussiert analysiert, wobei sowohl formale Aspekte als auch über die jeweilige Quelle anklingende Bedeutungsräume wichtig waren. Darüber hinaus wurde eine historische Kontextualisierung vorgenommen.

Im Folgenden werde ich zunächst einige konzeptuelle Gedanken zu Tod, Religion und Gender anführen, um danach am Beispiel von Trauerkleidung auf den Wandel der Inszenierung von Trauer in Relation zu Gendernormierungen zu fokussieren. Der Aufsatz wird mit einer synthetisierenden Generalisierung der gemachten Beobachtungen geschlossen.

\section{Tod, Religion und Gender: Ein aufschlussreicher Blickwinkel}

Das Dreigestirn Gender, Tod und Religion wird im Folgenden, wie erwähnt, in einen kulturgeschichtlichen Deutungsrahmen gestellt. Tod, Gender und Religion sind Begriffe, die menschliche Erfahrungen und Erlebnisse sinnhaft mit Bedeutung versehen und Differenzierungen zwischen verschiedenen Aspekten von Kultur schaffen. Dabei bildet die These, dass alle drei im Blick stehenden Dimensionen, also Tod, Gender und Religion, kulturelle Größen sind, die sich zeitund kulturspezifisch unterschiedlich ausformen, die Grundlage des hier vertretenen Ansatzes. Ich will diese kultur- und zeitspezifische Sichtweise aus Platzgründen nur am Tod erläutern - Ähnliches kann jedoch auch bezüglich Gender und Religion reflektiert werden. ${ }^{22}$

Was genau der Tod ist und ab wann eine Person als verstorben gilt, ist nicht zu jeder Zeit und in jedem soziokulturellen Umfeld identisch: ${ }^{23}$ Der Tod kann zum Beispiel körperlich mit einem Herzstillstand, mit dem Aufhören der Gehirnaktivität oder mit dem Zersetzen des Körpers verbunden werden, man denke für Letzteres etwa an die europäische Idee des Vampirismus, bei dem ein physisch im Grab nicht zersetzter Körper als ein Weiterleben gedeutet wird. Der Tod kann aber auch vom Körperlichen stärker gelöst werden. Verschiedene Forscher betonen die soziale Dimension des Todes ${ }^{24}$ wobei sogar von einem "sozialen Tod“

20 Zum Zugang: Burke 2009.

21 Vergleiche die zahlreichen Bilder bei Hauser 1994; Hoefer 2010.

22 Vgl. als erste Einführung zu Gender und Religion: King 1987; King 1995; King / Beattie 2004; Heller 2003; Höpflinger / Jeffers / Pezzoli-Olgiati 2008; Moser / Lanwerd 2010.

23 Vgl. Höpflinger 2015.

24 Vgl. bereits Hertz 1905 / 1906. 
die Rede sein kann: Menschen können sozial sterben im Sinne eines Verlustes der sozialen Pflichten, Rechte oder Anerkennungen, ohne jedoch körperlich tot zu sein, zum Beispiel bei Demenz oder im Kontext der Sklaverei. ${ }^{25}$ Weit verbreitet und zum Teil stark kulturprägend (man denke an das Alte Ägypten) ${ }^{26}$ ist auch die Idee, dass der Tod kein Ende, sondern eine Transformation in eine andere Existenz darstelle. Jenseitsvorstellungen zeugen von dieser Idee.

Dass der sinnhafte Umgang mit Sterben und Tod nicht nur bezüglich der Vermittlung solcher Jenseitsvorstellungen zu den grundlegenden Aufgaben von Religion gehört, wurde in unterschiedlichen Studien ausgeführt. ${ }^{27}$ Der Tod wurde verschiedentlich als eine der grundlegenden Fragen des Menschen gedeutet, der Umgang mit dem Sterben und mit Trauer als eine der Aufgaben religiöser Orientierungsschaffung verstanden. ${ }^{28}$ Beispielsweise definiert Fritz Stolz Religion als ein Orientierungssystem, das für den Menschen Unkontrollierbares (den Tod) in die Kontrolle überführt und einen Umgang damit ermöglicht (durch Vorstellungen, Rituale), aber dennoch das Problem an sich nicht löst. ${ }^{29}$ Religion vermittle so umfassenden Sinn. Auf einer empirischen Ebene bieten viele religiöse Traditionen Normen, Praktiken und Vorstellungen, um den Umgang mit eigenem und fremdem Sterben sinnhaft zu ermöglichen $;^{30}$ auch säkulare Bestattungsrituale sind oft von rituellen Handlungen (die aus religiösen Traditionen gespeist sind) oder von Jenseitsvorstellungen geprägt. Religion und der Umgang mit Tod können also in enger Wechselbeziehung zu einander gelesen werden. Weniger reflektiert ist dagegen der Einbezug von Gender in diese Relation. ${ }^{31}$ Gender, Religion und Tod sind auf verschiedene Weisen mit einander vernetzt; ich will zwei Wechselwirkungen, die eng miteinander verknüpft sind, hervorheben:

Erstens kann der Tod religiös auf einer repräsentativen Ebene personifiziert werden: beispielweise als Gevatter Tod oder als männlicher Fährmann (z. B. der griechische Charon). ${ }^{32}$ Damit wird dem Tod ein Gesicht gegeben, mit dem sich leichter umgehen lässt als mit etwas Unbekanntem. Bei dieser Personifizierung spielt Gender eine maßgebende Rolle, da je nach Geschlechtszuschreibungen andere Vorstellungswelten eröffnet werden. ${ }^{33}$ Der Tod kann wie soeben gesehen,

25 Sudnow 1973, S. 96-98; Goffmann 2006; zur Sklaverei als sozialer Tod: Patterson 1982.

26 Vgl. Assmann 2000.

27 Stellvertretend dafür Macho 2000.

28 Vergleiche als ersten Einblick: Stolz 2001; Assmann 2000; Macho 2000; Sörries 2009; Sörries 2012.

29 Vgl. Stolz 2001, S. 33.

30 Vergleiche als Beispiel Lüddeckens / Karanjia 2011.

31 Aufschlussreiche Werke dazu sind u. a.: Todd 1993; Guthke 1998; Kneuper 1999; Schroer 2014.

32 Vgl. Guthke 1998.

33 Vgl. ebd. 
männlich ausgeformt werden; er kann aber auch weiblich repräsentiert sein, man denke an weibliche Engel, wie sie auf Friedhöfen des 19. Jahrhunderts zu finden waren und Mitgefühl und Trauer symbolisierten, oder zuvor als weibliche Kadaver im Totentanz, die das Gender des/r Sterbenden reproduzierten oder gegenspiegelten. ${ }^{34}$ Soll der Tod allgemeiner als Ermahnung an jede Person stehen, finden sich Memento mori-Pärchen wie in Abb. 2. Bei dieser Abbildung handelt es sich um ein Klappbild aus den 1850er Jahren, das Tod, Gender und Religion explizit verbindet und mit einem normativen Programm ausstattet: Zugeklappt (Abb. 2, links) sieht man eine modische bürgerliche Dame und einen Herrn unter dem Spruch „Der Mensch von Erde ist gemacht, / Kleider sind nur Sündendecken / Was nützet denn die grosse Pracht / Heb sie nur auf du wirst erschrecken“. Öffnet man den Brief, wird ersichtlich, dass es sich bei beiden um Halbskelette handelt, die an die Unausweichlichkeit des Todes und damit zusammenhängend an ein bescheidenes und religiös korrektes Leben - auf der Hinterseite finden sich nämlich Abbildungen zu Sündenfall und Paradies - mahnen sollen.

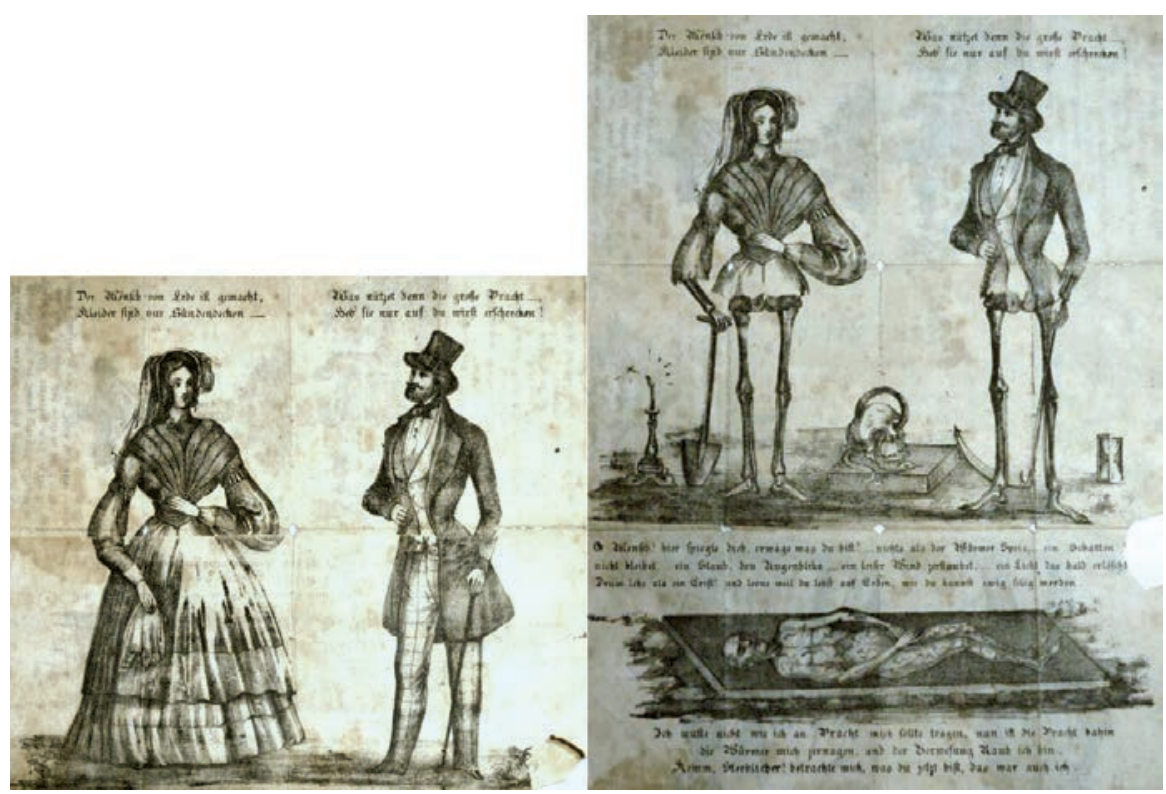

Abb. 2: Religiöser Klappbrief, Mitte 19. Jahrhundert, Privatbesitz. Foto: Höpflinger.

Aus solchen medial inszenierten normativen Forderungen wird eine zweite Dimension der Relation zwischen Gender und Tod gespeist, nämlich die sozioreli-

$34 \mathrm{Zu}$ weiblichen Engelfiguren auf Friedhöfen vergleiche Bulmer 2016; zu weiblichen Totentanzkadavern: Höpflinger 2015. 
giöse Ebene des regulierten praktischen Umgangs mit dem Tod und den Verstorbenen, auf den ich im Folgenden etwas genauer schauen will. Mein Blickwinkel setzt dabei bei der öffentlich-medialen Repräsentation von Trauer an.

Die öffentlich-mediale Inszenierung des Umgangs mit dem Tod war, wie das Eingangsbeispiel des Trauerzuges gezeigt hat, geschlechterspezifisch ausgeprägt. Gegenwärtig haben sich solche genderisierten Repräsentationen von Trauer verändert. Ich will deshalb auf den Wandel der Trauerinszenierung fokussieren und anhand des Beispiels der Trauerkleidung ausführen.

\section{Gehüllt in Krepp: Trauerkleidung im 19. Jahrhundert}

Medial, und damit zusammenhängend auch in der sozioreligiösen Praxis, ${ }^{35}$ war Trauer im 19. und frühen 20. Jahrhundert als vorwiegend weiblich repräsentiert. Die trauernde Frau als Grabesstatue auf Friedhöfen (Abb. 3), die trauernde Mutter mit Anlehnung an das Motiv der Pietà, die Frau am Grab als ikonographisches Motiv, die Klagefrau als Berufsidee, die betende Frau im Beinhaus auf Kupferstichen und in Geschichten - all dies sind Motive, die den Umgang mit dem Tod medial abbilden und kollektive Erwartungen gegenüber den Geschlechtern formen. ${ }^{36}$ Ein besonders herausstechendes Beispiel hierbei ist die Trauer als sozioreligiöser Status, der sich in christlich geprägten Trauernormen ausformt und in der Trauerkleidung abbildet. Ich will hierzu einige Gedanken vertiefen.

Das Inszenieren des Trauerstatus war im 18. Jahrhundert vom Adel ins Bürgertum übernommen worden. Im 19. Jahrhundert begann sich eine blühende bürgerliche Trauerkultur auszubilden, die besonderen Wert auf opulente Repräsentationen von Trauer legte und Hand in Hand ging mit der Industrialisierung. ${ }^{37}$ Sie wurde nicht durch staatlich festgelegte Gesetze, sondern durch sozioreligiöse Vorstellungen reguliert. Die christlichen Traditionen stellten dabei die normativen Grundlagen (Sünde, Verhüllung, Rückzug aus dem weltlichen Leben, Bescheidenheit, Aufopferung, Askese, etc.), die Trauerwarenhäuser die materielle Basis für die Markierung dieses Status zur Verfügung (Abb. 4). Den Normen entsprechende Trauer war materiell kostspielig, da zu einer gebührenden (d.h. die Verstorbenen ehrenden) Inszenierung, beginnend mit dem Sarg und dessen Ausstattung, dem Leichenwagen und den Pferden, dem Leidmahl, den

35 Für die enge Vernetzung zwischen kollektiven Vorstellungen, medialen Repräsentationen und der individuellen Praxis vergleiche: Pezzoli-Olgiati 2015.

36 Vergleiche zu weiblichen Grabesstatuen Michel 2002; Hoefer 2010; Bulmer 2016; zur Frau am Grab: Hoefer 2010; zur Klagefrau: Hauser 1994; zu betenden Frauen in Ossarien: Höpflinger / Müller 2016, S. 13, 67, 126.

37 Hoefer 2010. Wichtig für die Trauerkultur war beispielsweise der industriell in grossen Mengen hergestellte Krepp, eine künstlich stumpf gemachte Seide, die zum repräsentativen Stoff für Trauer wurde. 


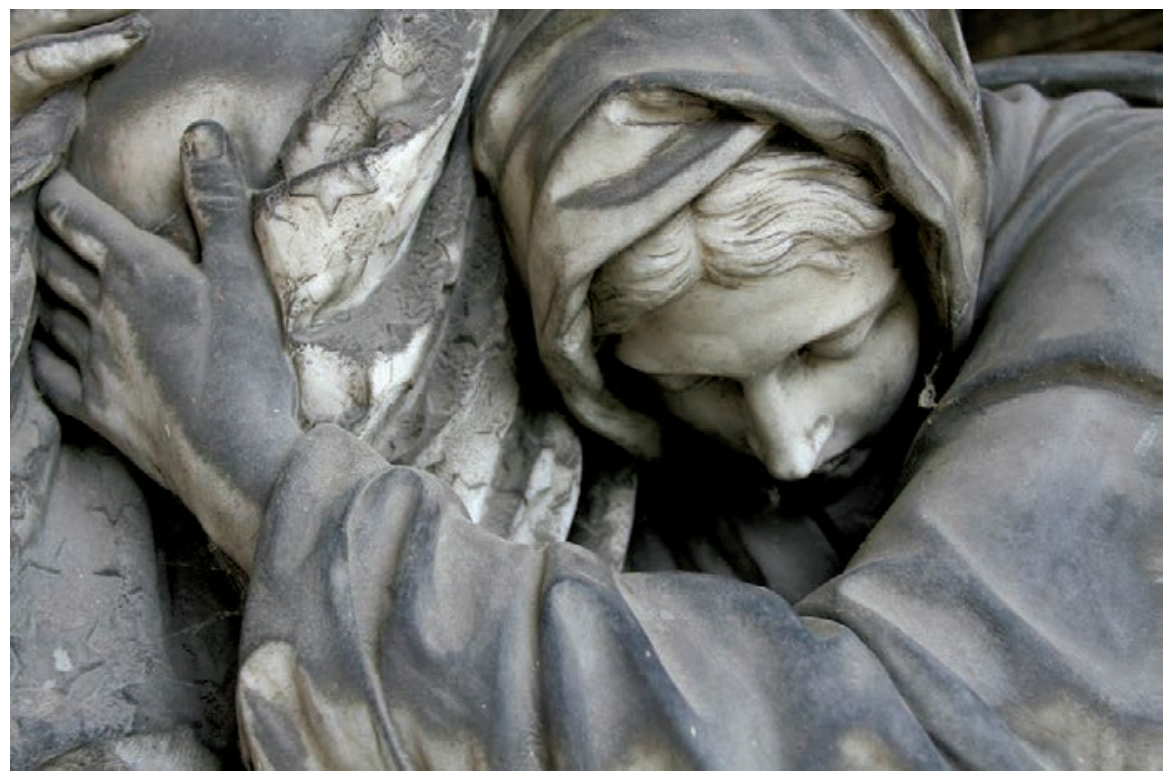

Abb. 3: Eine Trauerstatue auf dem Zentralfriedhof in Wien. Die idealisierte Trauernde umklammert eine mit einem Tuch bedeckte Urne. (C) Yves Müller.

Dekorationen, der Trauerkleidung etc., eine Menge an materiellen Gütern notwendig waren. Der Trauerstatus verpflichtete außerdem zu einer Auszeit aus der Gesellschaft, die man sich leisten können musste. ${ }^{38}$ Lou Taylor gibt für das Ende des 19. Jahrhunderts in Großbritannien als sozioreligiöse ideale Trauerzeit für eine Witwe um ihren Mann zweieinhalb Jahre Trauerzeit an, was voraussetzte, dass die Witwe sich in dieser Zeit in Schwarz kleidete, gesellschaftlich zurückzog, ihre religiösen Pflichten besonders wahrnahm und idealerweise auch keiner (bezahlten) Arbeit nachging. Dass sich dies z. B. eine trauernde Fabrikarbeiterin nicht oder nur in beschränktem Rahmen leisten konnte, liegt auf der Hand. ${ }^{39}$ Auffallend ist außerdem, dass Männer anderen Regulierungen unterlagen, wie ich weiter unten ausführen werde. ${ }^{40}$

38 Alleine eine angemessene Trauergarderobe für Frauen war ein kostspieliges Unternehmen, vergleiche Taylor 1983, S. 131 f: „Dresses of the early 1860s could include six to seven hundred yards of ribbon or trimmed edging".

39 In diesem Zusammenhang kam hinzu, dass der Verlust des Ehemanns für Frauen in einer Zeit, in der die Frau weder politisch noch bezüglich des Lohns gleichgestellt war, sozial und finanziell oft einschneidender war als der Verlust einer Ehefrau für einen Mann - man denke an die sprichwörtliche Verarmung von Witwen und Waisen.

40 Auch Kinder waren bezüglich Trauer noch nicht in dieser Art genderisiert. Zum Beispiel waren die Trauerkleider für kleine Kinder nicht geschlechterspezifisch. Vergleiche zu Kindern und Trauerkleidung: Taylor 1983, S. 164-187. 
Dieser Trauerstatus war nun, v.a. in Bezug auf Frauen, eng mit religiösen Normen und Vorstellungen konnotiert. Während des Trauerstatus sollte ein tugendhaftes religiöses Leben geführt werden; gesellschaftliche ,weltliche“ Vergnügungen waren zu meiden oder nur in geringen Mengen zu genießen $;{ }^{41}$ dem Gebet - im römisch-katholischen Raum für die armen Seelen - und der Besinnung sollte genügend Raum zugestanden werden. ${ }^{42}$

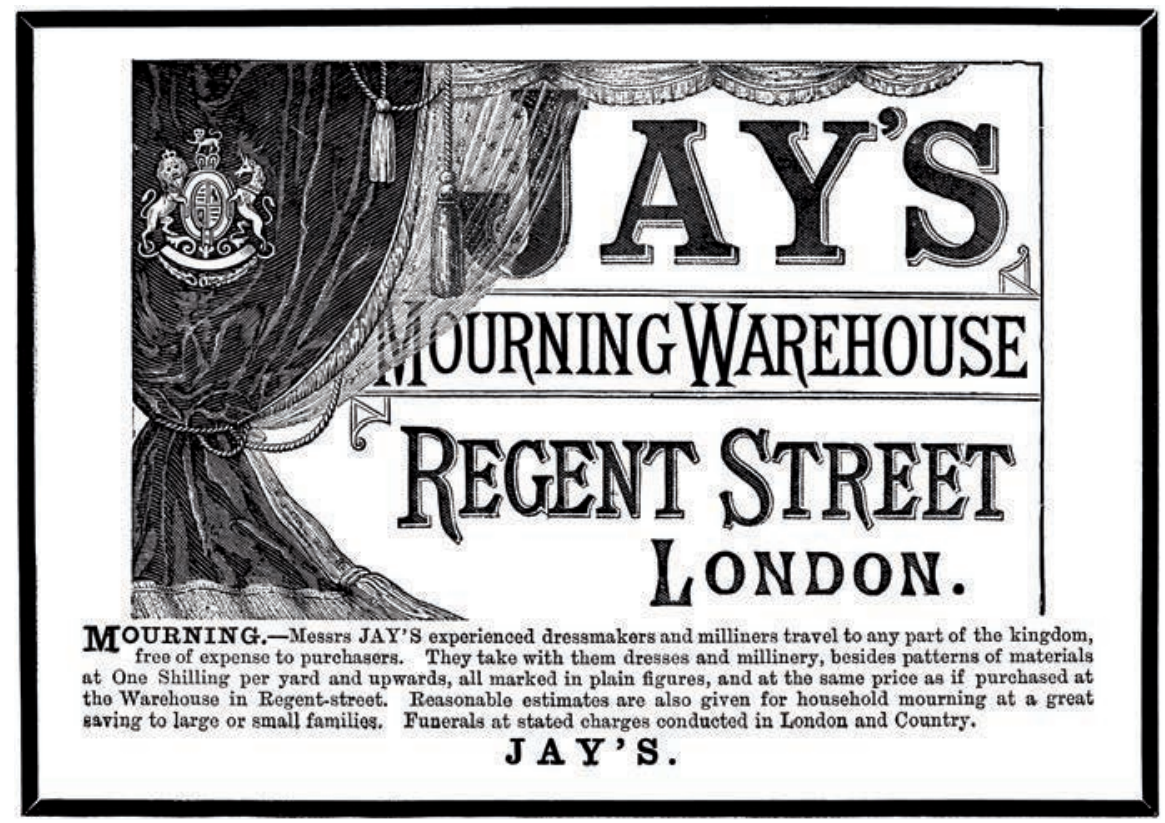

Abb. 4: Werbung für das Trauerwarenhaus „Jay’s“, London. Links ist ein Trauervorhang aus Krepp abgebildet.

Um diesen Trauerstatus und die Einhaltung der damit verbundenen Regeln gegen außen anzuzeigen, wurde im 19. Jahrhundert im Bürgertum Trauerkleidung immer wichtiger, wie Lou Taylor ausführt: „By the 1860s and 1870s, elegant mourning dress was a firmly established fact in the United States of America as well as in Europe“"43. Trauerkleidung war modisch und diente gleichzeitig den sie Tragenden als Erinnerung an die einzuhaltenden sozioreligiösen Regeln. Den Außenstehenden zeigte sie den spezifischen Status an und ermöglichte einen

41 Dass in der Praxis durchaus auch im Trauerstatus Bälle und andere gesellschaftliche Vergnügungen besucht wurden, zeigen Bilder von Trauerballkleidern, die bereits zu Beginn des 19. Jahrhunderts zu finden sind. Vgl. Hoefer 2010.

42 Vgl. Höpflinger / Müller 2016.

43 Taylor 1983, S. 132. 
adäquaten sozialen Umgang mit den Trägerinnen, aber auch eine Kontrolle über sie, damit das Trauern nicht durch ungemäßes Verhalten durchbrochen wurde.

Eine Klimax von Trauerkleidung lässt sich im letzten Drittel des 19. Jahrhunderts finden. ${ }^{44}$ In dieser Zeit wurden die sozioreligiösen Regeln hinsichtlich des Trauerverhaltens äußerst komplex: „As well as this vast increase in the use of mourning dress, the etiquette of mourning increased too. The mourning periods grew longer and even the remotest relatives were now mourned ${ }^{\text {“45. }}$. Dabei war dieses idealisierte Trauerverhalten stark geschlechtsspezifisch ausgeprägt: Trauern als emotionaler Ausdruck, aber vor allem die bürgerlichen Repräsentation des Trauerstatus war, wie oben bereits angedeutet, etwas Weibliches. Dies zeigt sich prägnant in der genderisierten Länge der idealen Trauerzeiten: Gemäß den Zahlen, die Lou Taylor für die Jahre zwischen 1876-1897 für Großbritannien rekonstruiert, sollte ein Mann für seine verstorbene Frau zu dieser Zeit idealerweise drei Monate Trauer tragen, eine Frau für ihren verstorbenen Mann jedoch ein Jahr und einen Tag Volltrauer und eineinhalb Jahre Halbtrauer. ${ }^{46}$ Aber nicht nur die auffallende Diskrepanz der Dauer, sondern auch die Art der Kleidung war für die Frau einschneidender als für den Mann: Während der Mann in dieser Zeit sozioreligiös lediglich „verpflichtet" war, schwarzen Trauerflor, meist in Form eines Trauerarmbandes (um den Oberarm geschlungen) zu tragen, ${ }^{47}$ sollte sich eine Frau - und besonders eine Witwe - im ersten Stadium der Volltrauer in der Öffentlichkeit in matten, vollkommen schwarzen Kleidern und vielfach verschleiert zeigen, so dass man von ihr idealerweise keinen Flecken Haut sah. Abb. 5 visualisiert diese Inszenierung: Mrs Howes, eine US-amerikanische Witwe hat für das Photoshooting die Kleidung angelegt, die sie bei der Bestattung trug und die sie in der ersten Phase der Volltrauer auch in der Öffentlichkeit tragen sollte, inklusive der Lederhandschuhe und des schwarzen Trauerschleiers.

Schmuck war - gemäß dem Ideal - abzulegen, oder sollte durch Trauerschmuck, dazu gehörte im beginnenden 19. Jahrhundert Eisengussschmuck ${ }^{48}$, später dann vor allem Jet-Schmuck und zum Teil Perlen, ersetzt werden. ${ }^{49}$ Die schwarze Trauerkleidung der bürgerlichen Frau war bis zum Ende der Volltrauerzeit zu tragen, wenn auch „nur“ noch mit Trauerhut und nicht mehr in Vollverschleierung (außer in gewissen Gegenden zur Kirche). In der Halbtrauer waren dann stufenweise weitere Farben zulässig: weiße Einsätze, graue und lila

44 Taylor 1983, S. 132 ff.

45 Taylor 1983, S. 133.

46 Vergleiche die Tabelle bei Taylor 1983, S. 303 f.

47 Ebd., S. 134: „Mourning dress for men in the nineteenth century, unlike that of their women, lessened, changing from the cloaked figure with trailing hat weepers to that of a normally dressed man distinguished only by a black armband".Vergleiche auch Hoefer 2010, S. 94.

48 Vergleiche dazu Höpflinger 2013.

49 Zu Trauerschmuck: Richter 2010; Hoefer 2010, S. 177-197. 


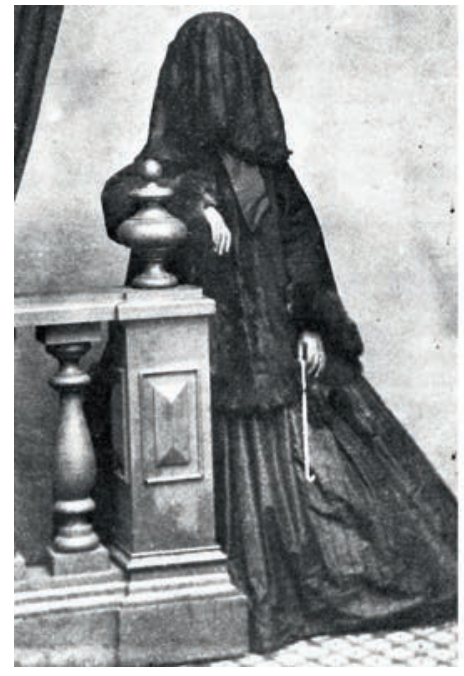

Abb. 5: Mrs Howes in Volltrauer, ca. 1860-70, USA.

oder violette Kleidung, vermehrt wieder Schmuck. ${ }^{50}$ Abb. 6 zeigt ein solches Halbtrauerkleid aus dem späten 19. Jahrhundert (USA): Es besteht aus schwarzem Brokat, der mit seiner Mischung aus glänzenden und matten Partien die Halbtrauer als Zwischenstatus zwischen der (matten) Volltrauer und dem normalen Alltag anzeigt. Ein weisser Einsatz mit Glasperlenornamenten in Grau und Lila bringt etwas Farbe ins Spiel.

Ein einschneidender Gender-Unterschied bezog sich auch auf die sozioreligiösen Normen des Wiederverheiratens nach dem Tod des/r Ehepartners/in: „Widowers, unlike widows, were able to remarry as soon as they pleased, even while still in mourning for their first wife ${ }^{\text {"51 }}$. Während eine Witwe sich während der zweieinhalb Jahre Trauer für ihren verstorbenen Mann also nicht wiederverheiraten sollte, unterlag der Witwer diesen Regulierungen nicht. Er konnte in der Trauerzeit arbeiten, wieder heiraten, sogar Vergnügungen nachgehen, ohne einen sozioreligiösen Prestigeverlust befürchten zu müssen.

Trauern hing also maßgebend mit genderspezifischen Erwartungen zusammen. Natascha N. Hoefer beschreibt Trauer als ,zweifelhaftes Privileg der Frauen“: Witwen und trauernde Frauen des Bürgertums und des Adels dienten ,als Statussymbole ihrer Familien “52. Eine Funktion der Inszenierung von Trauer war das Ausdrücken von Differenz. Hinzu kam, dass sich im 19. Jahrhundert „das Rollenklischee des vernunftgeleiteten Mannes, der außer Haus handelt und für seine Familie arbeitet, und der Frau, die als gefühlsgeleitetes Wesen der Engel von

50 Vgl. Taylor 1983; Hoefer 2010.

51 Taylor 1983, S. 133.

52 Hoefer 2010, S. 97. 


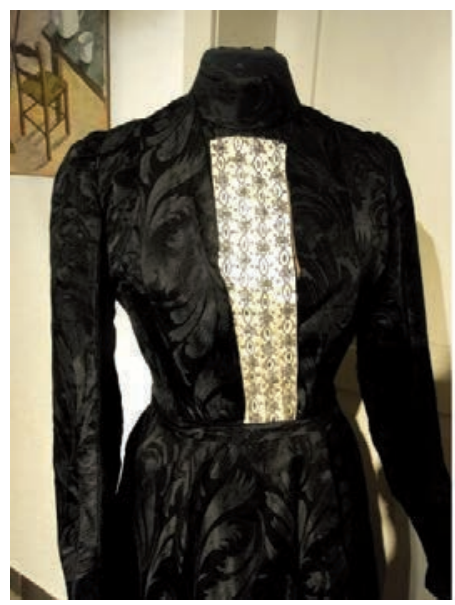

Abb. 6: Ein Kleid für die Halbtrauerzeit, spätes 19. Jahrhundert, USA. Privatbesitz. Foto: Höpflinger.

Heim und Herd ist ${ }^{* 53}$ verbreitete. Trauern wurde als etwas Emotionales gedeutet und in den Bereich der Frau geschoben. Der als rational gedachte Mann sollte im Falle der Trauer „seinen beherrschten Verlustschmerz mit verhaltenen Mitteln“ (also auch schlichter Trauerkleidung) anzeigen. ${ }^{54}$ „Die überbordende Trauer der Frau“ (die die Wichtigkeit und soziale Stellung des/r Verstorbenen spiegeln sollte) ,hingegen verlangte nach einer Fülle von schwarzem Stoff, je kostspieliger, umso repräsentativer ${ }^{655}$.

Die Repräsentation von Trauer verband also im 19. Jahrhundert die Idee eines der Frau entsprechenden emotionalen Naturells mit der Möglichkeit, sozialen Status anzuzeigen, ohne die religiösen Normen zu verletzen. Denn die Frau in üppiger Trauer war keineswegs eitel - die christliche Modekritik hatte sich immer vehement gegen weibliche Eitelkeit gerichtet ${ }^{56}$, , sondern sie opferte sich in einem moralisch verpflichtenden Sinn für ihre verstorbenen Verwandten, indem sie zu einer Leinwand für die Inszenierung von Trauer und Erinnerung wurde. Letztere machte die junge trauernde Witwe auch zu einem erotischen Motiv des 19. Jahrhunderts. ${ }^{57}$

Zu trauern war also ein „Privileg“ der bürgerlichen Frauen, das religiös untermauertes soziales Prestige schuf. Man konnte Reichtum anhand opulenter Trauer zeigen, ohne jedoch mit den religiösen Normen in Konflikt zu geraten. Im

53 Ebd.

54 Vgl. ebd.

55 Ebd.

56 Vergleiche zur christlichen Modekritik und der Verdammung von Eitelkeit: Wolter 2002.

57 Vgl. Hoefer 2010, S. 4-7. 
Gegenteil: Eine lange und vestimentär ausgiebige Inszenierung von Trauer (im Sinne strenger Verschleierung sowie expliziter Beachtung der Trauernormen) konnte als besonders religiös gedeutet werden. Hoefer spricht bezüglich der Verhüllung der Witwe von „,beständig nonnenhaft “58 . Der Körper der Frau wurde so zu einem Aushandlungsraum für die Formung sozioreligiöser Differenzen.

\section{4. „Bitte keine Trauerkleidung“‘: Der Wandel der Inszenierung von Trauer im 20. Jahrhunderts}

Die Bedeutung dieses vestimentär ausgedeuteten Trauerstatus wandelte sich im 20. Jahrhundert. Während sich in den 1920er Jahren noch ikonographische "Vorschriften“ für die ideale Kleidung der Trauernden in den verschiedenen Stadien der Repräsentation von Trauer finden lassen, ${ }^{59}$ sinkt die Bedeutung dieser Inszenierung ab Mitte des 20. Jahrhunderts, bis hin zu der seit den 1980er vielfach auf Todesanzeigen zu findenden Bitte, an der Bestattung nicht in Trauerkleidung zu erscheinen. Heute würde eine zweieinhalb Jahre andauernde vestimentäre und habituelle Inszenierung von Trauer durch eine Witwe eher Irritation als Verständnis oder gar Bewunderung hervorrufen. Sozioreligiöses Prestige ließe sich damit, zumindest in der europäischen Mainstreamgesellschaft, kaum mehr gewinnen.

Der Grund für diesen Wandel der Trauerrepräsentation ist multikausal: Die wirtschaftliche Lage veränderte sich besonders seit den 1950er Jahren und ermöglichte einer größeren Bandbreite von Menschen einen „diesseitsgerichteten“ Luxus. Massive medizinische Fortschritte nahmen vielen tödlichen Krankheiten ihren Stachel und professionalisierten und institutionalisierten den Umgang mit Sterbenden und dem Sterben. Politisch veränderten Demokratisierungsprozesse, gesellschaftlich die neuen Massenmedien (TV, später das Internet) die öffentliche Repräsentation von sozialen Hierarchien. Aber auch sich ändernde Gendervorstellungen bildeten eine Grundlage für diesen Wandel:

Einerseits ist es den feministischen und frauenrechtlichen Bewegungen zu verdanken, dass die Frau zunehmend aus ihrer Definition durch den Familienkontext gelöst wurde und als Individuum agieren konnte. Emanzipatorische Bestrebungen ermöglichten eine zunehmende Lösung aus dem Korsett der bürgerlichen Regulierung der Frau (und ihres Körpers), die im Hinblick auf den Nutzen für die Familie gesetzt waren. Die Aufgabe der Frau, durch ihren Körper (Schwangerschaften, vestimentäre Inszenierungen, Keuschheit im Witwenstatus, etc.) für soziales Prestige ihrer Verwandten und ihres lebenden oder toten Angetrauten zu sorgen, wurde zunehmend hinterfragt. In diesen emanzipatorischen Bewegungen spielte auch Religion auf verschiedenen Ebenen eine bedeutsame

58 Ebd., S. 67.

59 Vgl. Hoefer 2010, S. 112. 
Rolle. Beispielsweise findet sich das Erproben von neuen Lesarten und Frauenrollen innerhalb traditioneller Religionen, man denke an die Woman's Bible (publiziert in den Jahren 1895-1898). ${ }^{60}$ Andere Frauen suchten nach alternativen religiösen Ausdrucksformen, beispielsweise die weiblichen Medien im Spiritismus. ${ }^{61}$ Eine dritte feministische Möglichkeit bestand in der Forderung einer atheistischen Weltsicht, eine Vertreterin dieses Weges war Helene von Druskowitz (1858-1918). ${ }^{62}$ Dieser feministische Umgang mit Religion versuchte die Frau von als belastend empfundenen religiösen Pflichten wie den einengenden Trauerregeln zu befreien.

Andererseits wandelte sich im 20. Jahrhundert auch das bürgerliche Mainstream-Ideal der Frau: Die für ein weibliches Publikum ${ }^{63}$ medial ins Zentrum gesetzte idealisierte Frau des späten 19. Jahrhunderts war, wie gesehen, eine von religiösen Normen geformte, sich aufopfernde Figur, deren Schönheit sich in Bescheidenheit ausdrückte. Ab Mitte des 20. Jahrhundert wurde dieses (sich an ein weibliches Publikum gerichtetes) medial verbreitete Bild zu Gunsten einer Figur verändert, die sich zwar ebenfalls dem Mann unterordnete, aber den Körper ihm zu Gefallen inszenierte - und zwar zu seinen Lebzeiten. Die Jahrhundertelang von religiöser Seite kritisierte Eitelkeit ${ }^{64}$ wurde $\mathrm{zu}$ einem massenmedial vermittelten Ideal, das sich in der Gestalt des „Mannequins/ Fotomodells/ Models“65 ausprägte: Das Model war körperlich schön, erotisch, aufreizend und gleichzeitig eine weiße Leinwand für den (oft männlichen) Designer. In dieser Hinsicht der massenmedialen Repräsentation der idealen Frau findet sich also ein Wandel von einem religiös untermauerten Bild zu einem stärker von der Schönheitsindustrie propagierten Ideal. Die Trauer als Emotion blieb, aber die Repräsentation von Trauer als öffentlich-mediales Ideal (und damit auch die Erotisierung der jungen trauernden Witwe) wurde mehr und mehr durch die Inszenierung einer lebendigen Jugendlichkeit, die sich diesseits-gerichteten Werten hingab, ersetzt.

60 Jeffers 2008.

61 Crowley 2011.

62 Druskowitz 1988.

63 Die Idealisierung der Frau für ein männliches Publikum war vielschichtiger, dieses konnte auch Erotisierungen und Exotisierungen (man denke an Haremsbilder) in unterschiedlichen Schattierungen umfassen; vgl. Leitzke 2001, 19 f; Deeken/ Bösel 1996, S. 25; auch die junge Witwe konnte, wie oben erwähnt, erotisiert werden, vgl. Hoefer 2010.

64 Vgl. Wolter 2002.

65 Das Fotomodell gab es seit Erfindung der Fotografie, das Laufstegmodel seit Mitte des 19. Jahrhunderts, wobei die grossen Shows zu Beginn des 20. Jahrhunderts aufkamen. Zum sozialen Idealbild der Frau wurde dieser Beruf aber erst ab der Mitte des 20. Jahrhunderts. Vergleiche dazu: Evans 2013. 
Anna-Katharina Höpflinger

Aufschlussreich ist in diesem Kontext des Wandels der Trauerinszenierung zuletzt ein kurzer Blick auf die Wave Gothic-Szene: ${ }^{66}$ Diese in den 1980er Jahren aus der Punkbewegung hervorgegangene Subkultur charakterisiert sich durch eine neue Hinwendung zur Repräsentationen von Trauer - allerdings ohne den Anlass eines faktischen Todesfalls. Im visuellen Zentrum steht, nur grundlegend gesprochen, die Inszenierung eines - oft durchaus weiblichen - Umgangs mit Tod in einem medial-ästhetischen Sinn durch Kleidung, der Hauseinrichtung (ein Aspekt, der auch zu der Trauerkultur des 19. Jahrhunderts gehörte), Bilder, Texte. Es überrascht deshalb nicht, dass ein Teil dieser auch heute noch populären Subkultur sich an der sogenannten Viktorianischen Zeit sowie deren Inszenierung von Trauer orientiert und dass in dieser Szene religiöse Codes überraschend häufig aufgenommen werden. Die Repräsentation von Trauer und Religion interagieren auch hier, allerdings auf eine spezielle Art, die die damalige Todesrepräsentation ohne den faktischen Tod wiederbelebt.

\section{Wandel der Inszenierung von Differenz: Fazit}

Wie die bisherige Analyse gezeigt hat, formte und vermittelte die Repräsentation von Trauer soziale Grenzen. Ich will als Fazit drei solcher Grenzziehungsprozesse und ihre Transformation vom 19. zum 20. Jahrhundert hervorheben:

Erstens repräsentierte und erhärtete der Trauerstatus soziale Hierarchien, wie Abgrenzungen des Bürgertums (in Nachahmung des Adels) von der Arbeiterund Arbeiterinnenschaft. Der Trauerstatus formte sich in einer Art „Trauerluxus" aus, für den es im 19. Jahrhundert eine eigene Stoffart (Trauerkrepp) und ganze Warenhäuser gab (Abb. 4). Die Trauerkultur war ein wirtschaftlich bedeutsamer Zweig, der eng mit einem Selbstverständnis des Bürgertums zusammenhing und die Frau in einer religiös unproblematischen Art und Weise zur Repräsentantin von Luxus machte. Diese Inszenierung von Stand verschob sich durch die beiden Weltkriege des 20. Jahrhunderts und in der Nachkriegszeit, in denen die Hierarchien sich änderten und (zum Teil) eine soziale „Umschichtung“ der Bevölkerung stattfand, zu Gunsten einer Betonung säkularer Formen der Konstruktion sozialer Differenz. Es überrascht deshalb nicht, dass sich die Repräsentation von Trauer dort am längsten hielt, wo die „traditionellen“ Hierarchien am wenigsten Wandlung erfuhren, also in besonders ruralen Gebieten und im Adel.

Zweitens wurden im Trauerstatus dichotome Genderdifferenzen erhärtet: Die Trauerkultur war geschlechterspezifisch aufgebaut; für bestimmte Gender galten spezifische Regeln. Die Repräsentation von Trauer war in erster Linie etwas Weibliches, das verbunden wurde mit der Idee einer natürlichen Emotionalität der Frau. Trauern in der Idealform des 19. Jahrhunderts war eng an Erwartungen

66 Vergleiche als erste Einführung Brill 2008. 
gegenüber der Witwe und in einem etwas weiteren Sinn gegenüber Frauen allgemein geknüpft und basierte auf einer Idee der von Natur aus emotionalen Frau, die sich aufopfernd und aufgebend ihren Mann in liebender Erinnerung behalten und dies gegen außen anzeigen sollte. Ein Rollenvorbild hierfür bildete Königin Victoria, die bis an ihr Lebensende für ihren verstorbenen Prinzgemahl vestimentär trauerte. Die Idee der (vermeintlich) natürlichen Emotionalität der Frau wurde in den beiden Weltkriegen, in denen die Frauen in nahezu allen gesellschaftlichen Tätigkeitsbereichen ein Funktionieren sicherten ${ }^{67}$ aufgebrochen und kehrte in der Restauration der Geschlechterdifferenzierungen der 1950er Jahren nur kurz wieder zurück. Die Aufbrüche seit den 1968er Jahren konnten solche Ideen wirksam angreifen und neuen Rollenbildern von „Frau“ Raum schaffen. Das Aufgeben von Trauerrepräsentation kann also durchaus Frauenemanzipatorisch gelesen werden.

Trauerrepräsentation wurde - und das ist der dritte Punkt, den ich hervorheben will - mit normativen Vorstellungen verknüpft, die eng mit einem religiösen Weltbild verbunden waren. Auf der vestimentären Ebene ging es um Werte wie Bescheidenheit, Verzicht auf Kleidungsluxus und Erotik, Verhüllung und Zurückgezogenheit der eigenen Person (all das, was heute aus religiöser Sicht gerne unter dem Stichwort „modest dress“ abgehandelt wird). Die Trauerkleidung sollte also den sozioreligiösen Status und nicht die eigene Person in den Vordergrund stellen. Mit dieser Art der Inszenierung waren religiös-normative Handlungsmuster verbunden, die eine dienende, sich aufopfernde Rolle der Frau für ihren Mann voraussetzte und spiegelte: Die Witwe hatte für ihren Mann zu beten sowie ein besonders den religiöses Regeln entsprechendes Leben zu führen, um den verstorbenen Gemahl nicht postum mit Schande zu beflecken. Der Trauerstatus und die vestimentäre Repräsentation der Witwe kann also als eine bedeutende religiöse Verpflichtung ihrem verstorbenen Ehemann gegenüber verstanden werden. Die Veränderung dieser Pflicht ging einher mit einem Wandel der religiösen Normen, wie er sich vor allem seit den späten 1960er Jahren anbahnte. In diesen Jahrzehnten wurde die Suche nach persönlichen religiösen Erfahrungen, die durchaus in traditionellen religiösen Institutionen stattfinden konnte, stärker ins Zentrum gerückt, womit sich auch die auf einer kollektiven Regulierung basierte Inszenierung von Trauer wandelte. Trauerrepräsentation als ein Marker für religiöse Normen und Differenzierungen (z. B. „,korrekt“ - ,inkorrekt"; moralisch richtig oder falsch) traten hinter andere Formen religiöser Differenzbildung zurück (z. B. religiöse Erfahrung - keine religiöse Erfahrung).

Der Wandel der Repräsentation von Trauer kann, wie diese Beobachtungen zeigen, also aus einer genderzentrierten Sicht nicht als Verdrängung des Todes, sondern als eine Transformation von Grenzziehungsprozessen rekonstruiert werden: Der Wandel des Trauerstatus erweist sich als eine multidimensionale

67 Vergleiche als ersten Eindruck die Texte und v. a. das hervorragende Bildmaterial bei: Storey / Housego 2014 (erster Weltkrieg); Cassin-Scott 1980 (zweiter Weltkrieg). 
Verschiebung der Formung von Differenz, wobei sich ein enges Wechselspiel zwischen materieller Inszenierung, Gendervorstellungen sowie religiösen Normen und Weltbildern beobachten lässt. Denn die sozioreligiöse Repräsentation des Todes eröffnet nicht nur Erwartungen an das Individuum, sondern spiegelt, wie das Eingangsbeispiel der Beschreibung des Trauerzuges gezeigt hat, auch Vorstellungen bezüglich Hierarchien, Machtprozessen und kollektiver Handlungsformen.

\section{Literaturverzeichnis}

Assmann, Jan: Der Tod als Thema der Kulturtheorie. Todesbilder und Totenriten im alten Ägypten. Frankfurt am Main 2000.

Brill, Dunja: Goth Culture. Gender, Sexuality and Style. Oxford 2008.

Bulmer, Jane: Highgate Cemetery. London 2016.

Burke, Peter: What is Cultural History? Cambridge 2009.

Cassin-Scott, Jack: Women at War 1939-45. Oxford 1980.

Crowley, Karlyn: Feminism's New Age: Gender, Appropriation, and the Afterlife of Essentialism. New York 2011.

Deeken, Annette / Bösel, Monika: „An den süssen Wassern Asiens“. Frauenreisen in den Orient. Frankfurt am Main / New York 1996.

Druskowitz, Helene von: Der Mann als logische und sittliche Unmöglichkeit und als Fluch der Welt. Pessimistische Kardinalsätze. Hg. v. Hinrike Gronewold / Traute Hensch. Freiburg im Breisgau 1988.

Ellwanger, Karen / Helmhold, Heidi / Helmers, Traute / Schrödl, Barbara (Hgs.): Das „letzte Hemd“. Zur Konstruktion von Tod und Geschlecht in der materiellen und visuellen Kultur. Bielefeld 2010.

Evans, Caroline: The Mechanical Smile: Modernism and the First Fashion Shows in France and America, 1900-1929. New Haven 2013.

Fischer, Norbert: Vom Gottesacker zum Krematorium. Eine Sozialgeschichte der Friedhöfe in Deutschland. Köln 1996.

Fischer, Norbert: Geschichte des Todes in der Neuzeit. Erfurt 2001.

Goodwin, Sarah W.: Death and Representation. Baltimore 1993.

Goffman, Erwin: Asyle. Über die soziale Situation psychiatrischer Patienten und anderer Insassen. Stuttgart 2006.

Guthke, Karl S.: Ist der Tod eine Frau? Geschlecht und Tod in Kunst und Literatur. München 1998.

Hauser, Albert: Von den letzten Dingen. Tod, Begräbnis und Friedhöfe in der Schweiz 1700-1990. Zürich 1994.

Heierli, Julie: Die Volkstrachten von Zürich, Schaffhausen, Graubünden und Tessin. Erlenbach / Zürich 1930.

Heller, Birgit: „Gender und Religion“, in: Figl, Johann (Hg.): Handbuch Religionswissenschaft. Innsbruck-Wien / Göttingen 2003, S. 758-769.

Hertz, Robert: „Contribution à une étude sur la représentation collective de la mort“, in: L'Année sociologique (10) 1905/06, S. 48-137. 
Die Inszenierung von Trauer und der Wandel von Gendervorstellungen

Hoefer, Natascha N.: Schwermut und Schönheit. Als die Menschen Trauer trugen. Düsseldorf 2010.

Höpflinger, Anna-Katharina: „Eisen für Krieg und Trauer. Schmuck als Medium von Religion“, in: Glavac, Monika / Höpflinger, Anna-Katharina / Pezzoli-Olgiati, Daria (Hgs.): Second Skin. Körper, Kleidung, Religion. Göttingen 2013, S. 93-107.

Höpflinger, Anna-Katharina: „Gender Equality in Death? The Normative Dimension of Roman Catholic Ossuaries“, in: Religion and Gender (5/1) 2015, S. 18-34.

Höpflinger, Anna-Katharina / Müller, Yves: Ossarium. Beinhäuser der Schweiz. Zürich 2016.

Höpflinger, Anna-Katharina / Jeffers, Ann / Pezzoli-Olgiati, Daria (Hgs.): Handbuch Gender und Religion. Göttingen 2008.

Jeffers, Ann: „,Sapere Aude‘: Elizabeth Cady Stanton (1815-1902) and the Woman's Bible“, in: Höpflinger, Anna-Katharina / Jeffers, Ann / Pezzoli-Olgiati, Daria (Hgs.): Handbuch Gender und Religion. Göttingen 2008, S. 61-68.

King, Ursula: Women in the World's Religions. Past and Present. New York 1987.

King, Ursula: Religion and Gender. Oxford 1995.

King, Ursula / Beattie, Tina: Gender, Religion and Diversity. Cross-Cultural Perspectives. London / New York 2004.

Kneuper, Elsbeth: Tod, Weiblichkeit, Repräsentation. Forschen in einem deutschen Bestattungsunternehmen. Hamburg 1999.

Lanwerd, Susanne / Moser, Marcia Elisa (Hgs.): Frau-Gender-Queer. Gendertheoretische Ansätze in der Religionswissenschaft. Würzburg 2010.

Leitzke, Angelika: Das Bild des Orients in der französischen Malerei, von Napoleons Ägyptenfeldzug bis zum Deutsch-Französischen Krieg. Marburg 2001.

Lüddeckens, Dorothea / Karanjia, Ramiyar: Days of Transition. The Parsi Death Rituals. Göttingen 2011.

Macho, Thomas: „Tod und Trauer im kulturwissenschaftlichen Vergleich“, in: Assmann, Jan: Der Tod als Thema der Kulturtheorie. Todesbilder und Totenriten im alten Ägypten. Frankfurt am Main 2000.

Mäder, Marie-Therese: Die Reise als Suche nach Orientierung. Eine Annäherung an das Verhältnis zwischen Religion und Film. Marburg 2012.

Michel, Regula: Der Friedhof Sihlfeld in Zürich-Wiedikon. Bern 2002.

Nassehi, Armin / Weber, Georg: Tod, Modernität und Gesellschaft. Entwurfeiner Theorie der Todesverdrängung. Opladen 1989.

Patterson, Orlando: Slavery and Social Death - a Comparative Study. Cambridge, Massachusetts 1982 .

Pezzoli-Olgiati, Daria (Hg.): Religion in Cultural Imaginary. Explorations in Visual and Material Practices. Zürich / Baden-Baden 2015.

Richter, Isabel: „Erinnerungsspuren im Material. Trauerschmuck aus menschlichem Haar in der materiellen Kultur des Todes im 19. Jahrhundert“, in: Ellwanger, Karen / Helmhold, Heidi / Helmers, Traute / Schrödl, Barbara (Hgs.): Das „letzte Hemd“. Zur Konstruktion von Tod und Geschlecht in der materiellen und visuellen Kultur. Bielefeld 2010, S. 41-59.

Schroer, Silvia (Hg.): Sensenfrau und Klagemann. Sterben und Tod mit Gendervorzeichen. Zürich 2014.

Sörries, Reiner: Herzliches Beileid. Eine Kulturgeschichte der Trauer. Darmstadt 2012. 
Sörries, Reiner: Ruhe sanft. Kulturgeschichte des Friedhofs. Kevelaer 2009.

Stolz, Fritz: Grundzüge der Religionswissenschaft. Göttingen 2001.

Storey, Neil / Housego, Molly: Women in the First World War. Oxford 2010.

Sudnow, David: Organisiertes Sterben. Eine soziologische Untersuchung. Frankfurt am Main 1973.

Taylor, Lou: Mourning Dress. A Costume and Social History. London 1983.

Todd, Janet M.: Gender, Art and Death. Cambridge 1993.

Wolter, Gundula: Teufelshörner und Lustäpfel. Modekritik in Wort und Bild 11501620. Marburg 2002.

\section{Internetquellen}

Order of the Good Death, Website, verfügbar unter: www.orderofthegooddeath.com [01.06.2017].

\section{Abbildungsverzeichnis}

Abb. 1: Bilder aus Naters (VS, CH). Bild mit Zustimmung des Fotographen: (C) Yves Müller.

Abb. 2: Religiöser Klappbrief. Privatbesitz. Foto: Höpflinger.

Abb. 3: Eine Trauerstatue auf dem Zentralfriedhof in Wien, Bild mit Zustimmung des Fotographen; () Yves Müller.

Abb. 4: Werbung für das Trauerwarenhaus „Jay's“, London. Verfügbar unter: http://miss mary.com/household/1200-white-window-treatments-for-city-windows/ [28.04.2017].

Abb. 5: Mrs Howes in Volltrauer, ca. 1860-70, USA. Bild aus: Taylor 1983, S. 99.

Abb. 6: Ein Kleid für die Halbtrauerzeit, spätes 19. Jahrhundert, USA. Privatbesitz. Foto: Höpflinger.

Dr. Anna-Katharina Höpflinger, Religionswissenschaft und Religionsgeschichte, Evangelisch-Theologische Fakultät, Ludwig-Maximilians-Universität München, GeschwisterScholl-Platz 1, 80539 München, Germany, a.hoepflinger@lmu.de

Citation: Höpflinger, Anna-Katharina: „Schwarz, verhüllend, weiblich. Die Inszenierung von Trauer und der Wandel von Gendervorstellungen“, in: Heller, Birgit (ed.): Religion, Transformation and Gender. (J-RaT 2017 / 2), pp. 105-124.

Datum der Publikation: 02.10.2017 